These findings need to be interpreted with caution in view of the questionnaire used. The authors failed to ascertain whether the issue of doctors' dress was important to patients, prior to a specific choice of attire being made. Often, what doctors wear is not as big an issue for patients as we may believe and patients are reported to be less discriminating in their attitude towards physician appearance than physicians themselves (Dunn et al, 1987). Between 30 and $70 \%$ of patients in various studies are reported to have no preference regarding doctors' attire (Neinstein et al, 1985; Dunn et al, 1987; Friis \& Tilles, 1988; Del Rey \& Paul, 1995).

Patients should have first been asked whether their doctor's attire was of relevance to them, and those who felt it was could have gone on to choose one of the specified forms of dress. This study design would have provided a clearer picture of whether the issue of psychiatrists' dress was of significant concern to patients. If the majority felt that it was important, then the findings would have more relevance to clinical practice. To use an extreme example, consumers entering a fast food store may indicate a preference for one type of uniform over another when given two choices, but the majority may not really care as long as they get good service. While there are obvious differences between this situation and the issue of doctors' attire, the principle may well be the same.

Del Rey, J. A. G. \& Paul, R. I. (1995) Preferences of parents for paediatric emergency ptysician's attire. Paediatric Emergency Core. II. 361-364.

Dunn, J. J., Lee, T. H., Percelay, J. M., et al (1987) Patient and house officer attitudes on physician attire and etiquette. Journal of the American Medical

Association, 257, 65-68.

Fris, R. \& Tilles, J. (1988) Patients' preferences for resident physician dress style. Fomily Proctice Reseorch journal, 8. 24-31.

Glodhill, J. A., Warner, J. P. \& King, M. (1997) Psychiatrists and their patients: views on forms of dress and address. British journol of Psychiatry. 171. 228-232.

Neinstein, L. S., Stewart, D. \& Gordon, N. (1985)

Effect of physician dress style on patient - physician

relationship. Journol of Adolescent Heolth Core. 1, 456-459.

M. Rajagopalan Ballarat Health Services, PO Box 577, Ballarat, Victoria 3350, Australia

\section{Diagnostic criteria and functional psychosis}

Sir: The correspondence between Ryan (1997) and Van Os et al (1997b) made interesting reading. Van Os et al make an important claim that 'pure' affective and schizophrenic states become rarer with time. Strangely enough, their claim is not borne out by the results of a previous study they published (Van Os et al, 1996). In that study, the same sample when diagnosed with DSM-III-R diagnostic criteria led to a diagnosis of only 12 cases of schizoaffective psychosis, with schizophrenia being more or less stable, and 43 cases of affective psychosis, compared with 17 in their present study (Van Os et al, 1997a).

This raises the following questions. First, are the research diagnostic criteria (RDC) unreliable at distinguishing 'pure' forms of affective and schizophrenic psychosis? Second, is it only affective disorder that presents with schizophrenic symptoms over a lifetime (theoretically, the lack of pure forms should affect both diagnoses)? Third, if this were true of the RDC, should they be avoided in favour of DSM-III-R or any other reliable criteria used to diagnose schizophrenia and affective disorder?

Ryan, A. (1997) Psychopathological syndromes and familial morbid risk of psychosis (letter). British journal of Psychiatry, 171. 289.

Van Os, J., Faly, T. A., Jones, P., et al (1996) Psychopathological syndromes and the functional psychoses: associations with course and outcome. Psychological Medicine, 26, 161-176.

_, Marcelis, M., Sham, P. et ol (1997o)

Psychopathological syndromes and familial morbid risk of psychosis. British journal of Psychiatry. 170. 241-246.

_, - , et al (1997b) Psychopathological syndromes and familial morbid risk of psychosis (letter). British journol of Psychiotry, 171, 289.

D. Kirby Department of Psychiatry, Charing Cross and Westminster Medical School, London W6 8RP

Authors' reply: What Dr Kirby notes, but may not realise, is that given a mixture of affective and psychotic symptoms, it is a lot more difficult to get a diagnosis of schizoaffective disorder using DSM criteria than it is using the RDC. The DSM requirement that there must have been delusions or hallucinations for at least two weeks in the absence of prominent mood symptoms is simply more restrictive. We recently examined 706 patients with functional psychosis and found the same relative diagnostic shift between the RDC and DSM systems, with ICD-10 being somewhere in between. Thus, more patients with affective and psychotic symptoms will be labelled as suffering with affective psychosis according to DSM and with schizoaffective psychosis according to RDC.
We fail to see what this relative shift has to do with our statement that patients with psychosis accumulate a variety of affective and non-affective psychopathologies as time progresses, which will affect the diagnostic distribution within a given diagnostic system accordingly. The only way to examine this longitudinal issue is to compare baseline with follow-up diagnoses within the same diagnostic system.

The choice of diagnostic system (and the ensuing diagnostic distribution) is arbitrary because there is no evidence that any system is more valid than the other. Thus, our response to Dr Kirby's first question ("are the RDC unreliable at distinguishing 'pure' forms of affective and schizophrenic psychosis?") is that, for the time being, all diagnostic systems remain equally (un)reliable. In our 1997 paper (Van Os et al, 1997) we chose the RDC to diagnose the patients because of its compatibility with the family history research diagnostic criteria method used to diagnose the relatives. In our earlier paper (Van Os et al, 1996) we examined a clinical issue and therefore used both DSM and ICD criteria, because these are most often used in clinical practice. The question following from Dr Kirby's observation is: if there is no agreement between diagnostic systems as to where to draw the line between basic categories in the functional psychosis, should we not, instead, concentrate more on overlapping psychopathological dimensions within the continuum of psychosis?

Van Os, J., Fahy, T. A., Jones, P., et al (1996) Psychopathological syndromes and the functional psychoses: associations with course and outcome Psychologicol Medicine, 26, 161-176.

—, Marcelis, M., Sham, P., et ol (1997)

Psychopathological syndromes and familial morbid risk of psychosis. British journol of Psychiatry, 170. 241-246.

J. Van Os, M. Marcelis, P. Sham, P. Jones, K. Gilvarry, R. Murray Psychiatric Epidemiology Program. University of Maastricht, PO Box 616. 6200 MD Maastricht. The Netherlands

\section{Family involvement in the care of people with psychoses}

Sir: Should communication between psychiatrist and non-professional carers be permitted without the patient's agreement? Szmukler \& Bloch (1997) have confirmed my impression that the profession is at sea over this question. Community care of people with psychoses 